\title{
BMJ Open Disease burden of liver cancer in China from 1997 to 2016: an observational study based on the Global Burden of Diseases
}

Cheng Ding, ${ }^{1}$ Xiaofang Fu, ${ }^{1}$ Yuqing Zhou,,${ }^{1}$ Xiaoxiao Liu, ${ }^{1}$ Jie $\mathrm{Wu},{ }^{1}$
Chenyang Huang, ${ }^{1}$ Min Deng, ${ }^{1}$ Yiping $\mathrm{Li},{ }^{2}$ Lanjuan Li, ${ }^{1}$ Shigui Yang ${ }^{1}$

To cite: Ding C, Fu X, Zhou Y, et al. Disease burden of liver cancer in China from 1997 to 2016: an observational study based on the Global Burden of Diseases. BMJ Open 2019;9:e025613. doi:10.1136/ bmjopen-2018-025613

- Prepublication history and additional material for this paper are available online. To view these files, please visit the journal online (http://dx.doi. org/10.1136/bmjopen-2018025613).

CD and XF contributed equally.

Received 30 July 2018 Revised 25 November 2018 Accepted 7 March 2019

Check for updates

(c) Author(s) (or their employer(s)) 2019. Re-use permitted under CC BY-NC. No commercial re-use. See rights and permissions. Published by BMJ.

${ }^{1}$ State Key Laboratory for Diagnosis and Treatment of Infectious Diseases, Collaborative Innovation Center for Diagnosis and Treatment of Infectious Diseases, The First Affiliated Hospital, College of Medicine, Zhejiang University, Hangzhou, Zhejiang, China ${ }^{2}$ Digital medicine, Zhejiang Institute of Medical Care Information Technology, Hangzhou, Zhejiang, China

Correspondence to Professor Lanjuan Li; |jli@zju.edu.cn and Dr Shigui Yang;

YANGSHIGUI@zju.edu.cn

\section{ABSTRACT}

Objective To evaluate the trends in disease burden and the epidemiological features of liver cancer in China while identifying potential strategies to lower the disease burden. Design Observational study based on the Global Burden of Diseases.

Participants Data were publicly available and deidentified and individuals were not involved.

Measurement and methods To measure the liver cancer burden, we extracted data from the Global Health Data Exchange using the metrics of prevalence, incidence, mortality and disability-adjusted life years (DALYs). Joinpoint and negative binomial regressions were applied to identify trends and risk factors.

Results From 1997 to 2016, the prevalence, incidence, mortality and DALYs of liver cancer in China were from 28.22/100 000 to $60.04 / 100000$, from $27.33 / 100000$ to $41.40 / 100000$, from 27.40/100 000 to $31.49 / 100000$ and from 10311308 to 11539 102, respectively. The prevalence, incidence and mortality were increasing, with the average annual percent changes (AAPCs) of $4.0 \%$ (95\% Cl 3.9\% to $4.2 \%), 2.1 \%$ (95\% Cl $2.0 \%$ to $2.2 \%$ ) and $0.5 \%$ (95\% Cl $0.2 \%$ to $0.9 \%)$, respectively. Meanwhile, the rate of DALYs was stable with the AAPCs of $-0.1 \%$ (95\% $\mathrm{Cl}-0.4 \%$ to $0.3 \%)$. The mortality-to-incidence ratio of liver cancer decreased from 1.00 in 1997 to 0.76 in 2016 $(\beta=-0.014, p<0.0001)$. Males (OR: $2.98,95 \% \mathrm{Cl} 2.68$ to 3.30 for prevalence, OR: $2.45,95 \% \mathrm{Cl} 2.21$ to 2.71 for incidence) and the elderly individuals (OR: $1.57,95 \% \mathrm{Cl}$ 1.55 to 1.59 for prevalence, OR: $1.58,95 \% \mathrm{Cl} 1.56$ to 1.60 for incidence) had a higher risk. Hepatitis $\mathrm{B}$ accounted for the highest proportion of liver cancer cases $(55.11 \%)$ and deaths (54.13\%).

Conclusions The disease burden of liver cancer continued to increase in China with viral factors as one of the leading causes. Strategies such as promoting hepatitis $B$ vaccinations, blocking the transmission of hepatitis $C$ and reducing alcohol consumption should be prioritised.

\section{INTRODUCTION}

Cancer results from interactions between genetic risk factors and external agents and is one of the leading causes of death worldwide. ${ }^{1}$ Liver cancer is the sixth most common cancer
Strengths and limitations of this study

- A long-term period (20 years) on the trend of liver cancer burden in China was evaluated in this study.

- Disease burden trends and the associated risk factors of liver cancer were quantified to reveal the extent of the epidemic and suggest potential strategies against liver cancer.

- The findings might be limited by the fact that the data used in this study were secondary estimated data rather than the original epidemic or surveillance data.

worldwide ${ }^{2}$ and the second common cause of cancer-related death, and which accounted for 788000 deaths in 2015. ${ }^{1}$ Hepatocellular carcinoma (HCC) is the most common type of liver cancer, while intrahepatic cholangiocarcinoma is a rarer form of the disease. ${ }^{3} \mathrm{~A}$ wide range of environmental risk factors have been associated with liver cancer, such as viral factors (hepatitis B and hepatitis $\mathrm{C}$ ) and non-viral factors (alcohol, aflatoxin, non-alcoholic fatty liver disease, obesity, diabetes and smoking) ${ }^{4-8}$ Reducing exposure to these risk factors is the most effective way to minimise the risk of developing liver cancer.

Previous studies show that the disease burden of liver cancer is increasing and that it is not equally distributed throughout the population. ${ }^{9}$ Incidence rates are elevated in East and South-East Asia, Africa and Melanesia, with nearly three-quarters of new liver cancer cases occurring in regions of low or medium development. ${ }^{3}$ In China, the reported averages for the annual incidence and mortality of liver cancer between 2003 and 2007 were approximately 26.68/100 000 and 25.03/100 000, respectively. ${ }^{10}$ In 2015, China accounted for over half of the worldwide liver cancer incident cases (446 100 
cases) and deaths (422 100 cases). ${ }^{11}$ Historically, liver cancer has been the most prevalent malignancy in China.

The updated global action plan from 2013 to 2020 for the prevention and control of non-communicable diseases issued by the WHO aims to address four major categories of disease (cardiovascular disease, diabetes, chronic respiratory disease and cancer), with the goal of achieving a $25 \%$ reduction in premature mortality by $2025 .{ }^{12}$ Given the massive population in China, the availability of study sample sites for estimating incidence and mortality was limited (308 sites in 2014). ${ }^{11}$ As a result, comprehensive long-term assessments of liver cancer burden among larger populations at the national level are required.

To address this need, this study provides an overview of the trends in the disease burden of liver cancer during the past two decades in China while identifying the factors that contribute the most to declining health caused by liver cancer. By analysing trends and identifying risk factors for liver cancer, we hope to reveal the extent of the epidemic and suggest potential strategies to lower its disease burden in China.

\section{METHODS}

\section{Study design}

The Global Burden of Disease (GBD) Study 2016 incorporated data from a large number of sources to estimate mortality, causes of death and illness and risk factors in 195 countries and territories from 1990 to $2016 .{ }^{13}$ The GBD 2016 used multiple methods to integrate a large number of available data sources with the specific epidemiology of each disease, and the Bayesian meta-regression tool DisMod-MR V.2.1 was used as the main method of estimation. ${ }^{14}$ Detailed information about data resources, definitions, statistical modelling and efforts to improve data quality have been previously reported. ${ }^{14-17}$

We extracted GBD 2016 data on liver cancer in China from 1997 to 2016 using the Global Health Data Exchange. ${ }^{13}$ The variables obtained from these data included year, age, gender and cause (hepatitis B, hepatitis $\mathrm{C}$, alcohol use and other causes). The incidence, prevalence, mortality and disability-adjusted life years (DALYs) were used as the primary metrics to assess the impacts on health within population. The total number of liver cancer cases and rates of each metrics were provided by the GBD 2016. Prevalence rate (per 100 000) was defined as aggregated cases (including new cases and previously diagnosed cases) divided by the population size; incidence rate (per 100 000) was defined as the number of new cases divided by the population size; mortality rate (per 100000 ) was defined as the number of annual deaths divided by the total population size and DALYs were summed by the years of life lost and the years of life lived with disability. The age-adjusted rates (ARs) for each metric were adjusted to population of China in $2010 .{ }^{18}$ The mortality-to-incidence (MI) ratio was used as an indicator of disease severity and the efficacy of clinical treatment and management.

\section{Patient and public involvement}

Patients or public were not involved in the recruitment and conduct of this study.

\section{Statistical analysis}

The average annual percent change (AAPC) and annual percent change (APC) were used to evaluate trends in disease burden and calculated using the Joinpoint Regression Program (V.4.5.0.1, June 2017). The values of AAPC and APC were calculated as $[(\operatorname{Exp}(b i)-1)] \times 100$, where bi represents the slope of the period segment. The $\mathrm{Z}$ test was used to assess whether an AAPC or APC was different from zero. The terms 'increase' and 'decrease' were used when the slope (AAPC or APC) of the trend was statistically significant, while 'stable' referred to a non-significant trend. A negative binomial regression model was utilised to identify potentially influential factors using the SAS V.9.4 software. The OR and its $95 \%$ CI were also calculated for each factor. Origin 2017 software (MicroCal Software, Northampton, Massachusetts, USA) was used to draw the figures. The significance level for the hypothesis tests was set at 0.05 .

\section{RESULTS}

The number of liver cancer patients increased from 349127 (prevalence: 28.22/100 000, AR: 38.64/100 000 ) in 1997 to 814026 (prevalence: 60.04/100 000, AR: 53.18/100 000) in 2016. The number of new cases increased from 338063 (incidence: 27.33/100 000, AR: 37.55/100 000) to 561249 (incidence: 41.40/100 000, AR: 36.62/100 000). There were 338904 deaths (27.40/100 000, AR: 38.03/100 000) in 1997 and 426 900 deaths (31.49/100 000, AR: $27.73 / 100$ 000) in 2016. The DALYs also increased from 10311308 (rate: $833.52 / 100$ 000, AR: $1104.65 / 100000$ ) in 1997 to 11 539102 (rate: 851.16/100 000, AR: 759.02/100 000) in 2016 (figure 1 and online supplementary table 1 ). There were increasing trends from 1997 to 2016 in prevalence (AAPC: $4.0 \%, 95 \%$ CI $3.9 \%$ to $4.2 \%$ ), incidence (AAPC: $2.1 \%, 95 \%$ CI $2.0 \%$ to $2.2 \%$ ) and mortality (AAPC: $0.5 \%$, $95 \%$ CI $0.2 \%$ to $0.9 \%$ ), while the rate of DALYs remained stable (AAPC: $-0.1 \%, 95 \% \mathrm{CI}-0.4 \%$ to $0.3 \%$ ). The APC increased for prevalence since 2009, for incidence since 2010, and for mortality and rate of DALYs since 2013 (table 1 and online supplementary figure 1).

Increasing trends in prevalence, incidence and mortality were seen among males. Increasing trends in prevalence and incidence were seen among females, while a decreasing trend was observed in rate of DALYs (AAPC: $-0.7 \%, 95 \%$ CI $-1.1 \%$ to $-0.3 \%$ ). The AAPCs in prevalence, incidence, mortality and rate of DALYs among males were higher than those among females (online supplementary table 1 ). The male to female ratio ranged from 3.05 to 3.98 in prevalence, 2.67 to 3.27 in incidence, 2.54 to 2.96 in mortality and 2.93 to 3.44 in DALYs during the observation period (figure 2). 

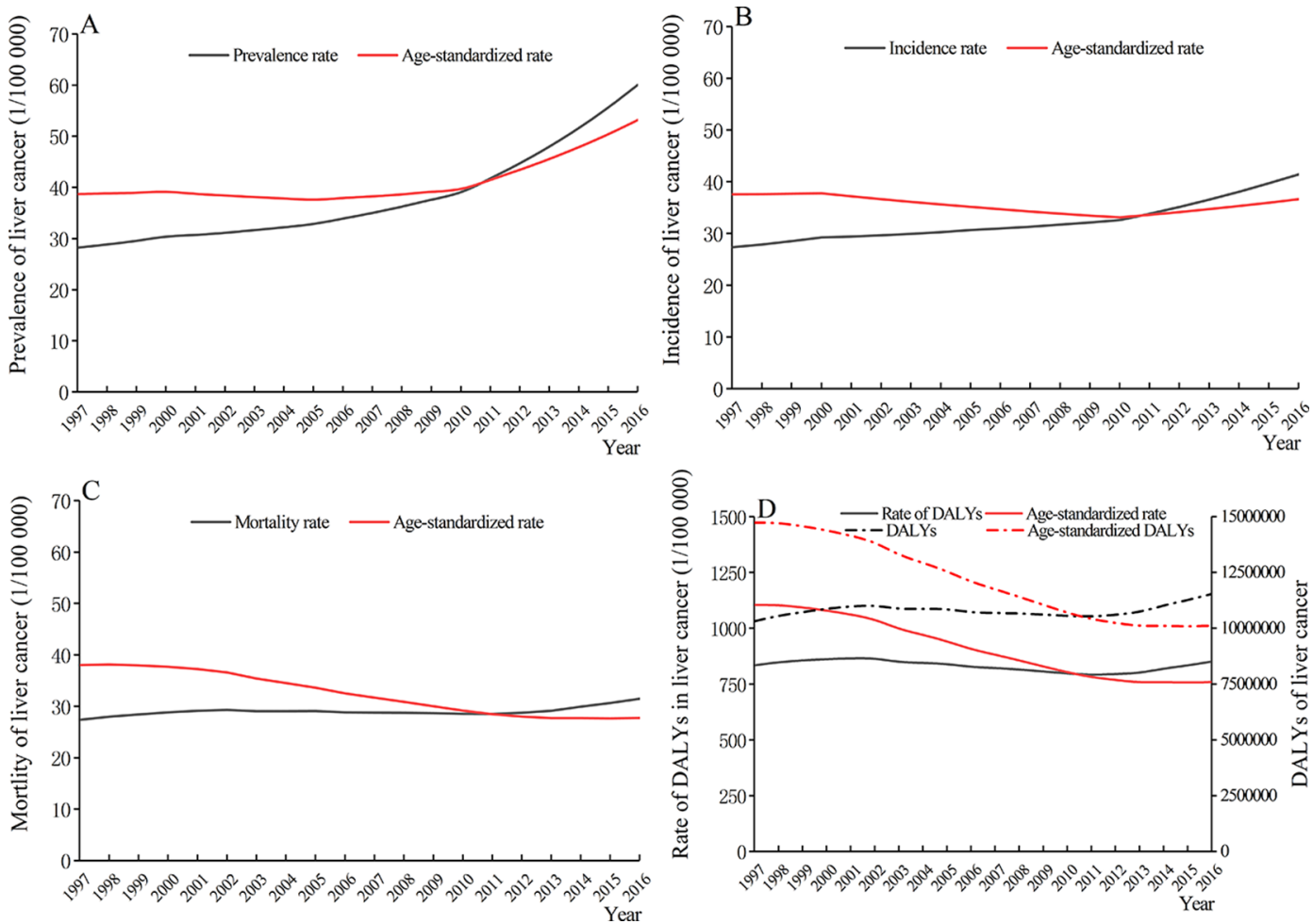

Figure 1 Observed and age-adjusted rates (ARs) of liver cancer from 1997 to 2016 in China. (A) The prevalence and ARs of liver cancer; (B) the incidence and ARs of liver cancer; (C) the mortality and ARs of liver cancer; (D) the observed and ageadjusted DALYs and rates of DALYs of liver cancer. DALYs, disability-adjusted life years.

Figure 3 shows the rates of liver cancer among different age groups. The prevalence was under 1.00/100 000 for individuals aged below 20 and increased rapidly between the ages of 40 and 70 . The highest prevalence rate was observed in those aged 80-84. Similar tendencies were observed in incidence and mortality with rates that were

Table 1 The trends in liver cancer burden by joinpoint regressions

\begin{tabular}{llll}
\hline Metric & Index & Period & $\begin{array}{l}\text { Estimate } \% \\
\mathbf{( 9 5 \%} \mathbf{C l})\end{array}$ \\
\hline Prevalence & APC & $1997-2009$ & $2.2(2.0 \text { to } 2.4)^{\star}$ \\
& APC & $2009-2016$ & $7.2(6.9 \text { to } 7.6)^{\star}$ \\
Incidence & AAPC & $1997-2016$ & $4.0(3.9 \text { to } 4.2)^{\star}$ \\
& APC & $1997-2010$ & $1.2(1.1 \text { to } 1.3)^{\star}$ \\
Mortality & APC & $2010-2016$ & $4.1(3.8 \text { to } 4.3)^{\star}$ \\
& AAPC & $1997-2016$ & $2.1(2.0 \text { to } 2.2)^{\star}$ \\
\hline Rate of DALYS & APC & $1997-2013$ & $0.1(-0.1$ to 0.3$)$ \\
& APC & $2013-2016$ & $2.8(0.6 \text { to } 5.1)^{\star}$ \\
& APC & $1997-2016$ & $0.5(0.2 \text { to } 0.9)^{\star}$ \\
\hline & AAPC & $1997-2013$ & $-0.5(-0.7 \text { to }-0.3)^{\star}$ \\
& APC 2016 & $-0.1(-0.4$ to 0.3$)$ \\
\hline
\end{tabular}

${ }^{*} \mathrm{P}<0.05$.

AAPC, average annual percent change; APC, annual percent change; DALYs, disability-adjusted life years. consistently high among those aged over 90 . A higher rate of incidence than that of prevalence was also observed among those aged over 90 . The lowest rate of DALYs was over 1500/100 000 among those aged 50-84 which indicates that older individuals experience a heavier disease burden from liver cancer.

The MI ratios were consistently high, with the lowest ratio of 0.74 occurring in males during 2016. Overall, the MI ratio decreased from 1.00 in 1997 to 0.76 in 2016 $(\beta=-0.014, p<0.0001)$, with an annual rate of change of $-1.44 \%$. The annual rates of change in the MI ratio for males and females were $-1.50 \%$ and $-1.23 \%$, respectively (online supplementary table 2). Additionally, the MI ratios showed decreasing trends in both males $(\beta=-0.012$, $\mathrm{p}<0.0001)$ and females $(\beta=-0.014, \mathrm{p}<0.0001)$, but there was no significant trend in magnitude in these two groups $(\mathrm{t}=-1.64, \mathrm{p}=0.109)$ (figure 4$)$.

The percentages of liver cancer cases attributed to alcohol use, hepatitis $\mathrm{B}$, hepatitis $\mathrm{C}$ and other causes were approximately $10 \%, 55 \%, 8 \%$ and $27 \%$, respectively, and each of these metrics remained relatively stable during this period. Hepatitis B accounted for $55.11 \%$ of liver cancer cases and $54.13 \%$ of liver cancer deaths in China and showed a slightly downward trend (online supplementary table 3). Differences in the distributions of live cancer causes between age groups were observed. For example, the proportions of causes related to alcohol use and hepatitis $\mathrm{C}$ increased with age (online supplementary table 4$)$. 

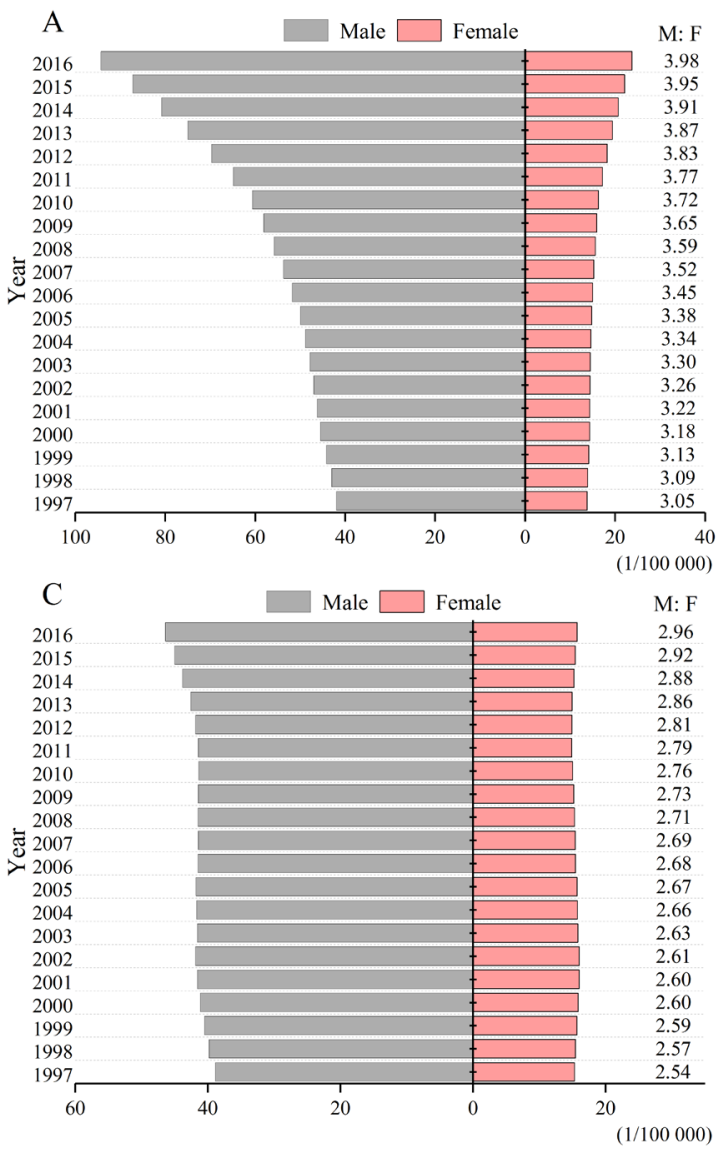
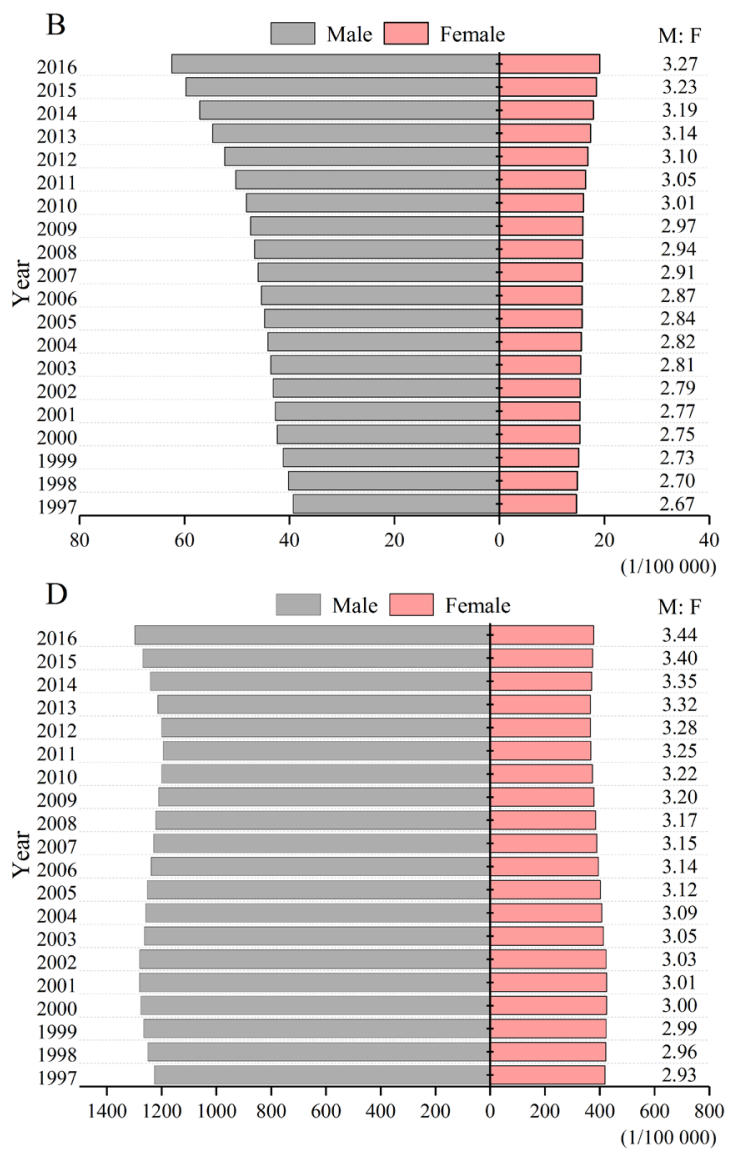

Figure 2 Disease burden of liver cancer among different gender groups from 1997 to 2016 in China. (A) The differences in prevalence between males and females; (B) the differences in incidence between males and females; (C) the differences of mortality between males and females; (D) the differences in DALYs between males and females. DALYs, disability-adjusted life years.

Since the results of Lagrange multiplier test were significant for all metrics (all $\mathrm{p}<0.0001$ ), negative binomial regressions were applied for the risk factors analysis of the scaled dataset (table 2). The male odds for prevalence (OR: 2.98, 95\% CI 2.68 to 3.30), incidence (OR: $2.45,95 \%$ CI 2.21 to 2.71 ), mortality (OR: $2.36,95 \%$ CI 2.15 to 2.59 ) and rate of DALYs (OR: $2.46,95 \%$ CI 2.16 to 2.80) were more than two times higher than those of females. The fold change in ORs for every 5 -year increase in age were 1.57 (95\% CI 1.55 to 1.59 ) for prevalence, 1.58 (95\% CI 1.56 to 1.60$)$ for incidence, 1.57 (95\% CI 1.55 to 1.59$)$ for mortality and $1.42(95 \%$ CI 1.39 to 1.44$)$ for rate of DALYs. Hepatitis B and other causes led to a higher risk than alcohol use with the ORs ranging from 2.28 to 5.63 in prevalence, incidence, mortality and rate of DALYs $(\mathrm{p}<0.0001)$.

\section{DISCUSSION}

\section{The disease burden of liver cancer in China}

The number of new cancer cases is expected to exceed 20 million globally by 2025 , compared with 14.1 million cases in 2012. ${ }^{3}$ In China, there were 4292000 new cancer cases and 2814000 cancer deaths estimated in $2015 .{ }^{11}$ We found that the prevalence, incidence, mortality and DALYs of liver cancer increased from 1997 to 2016, which indicates an increasing disease burden in China. Additionally, higher APCs in all metrics were observed in recent years. Similar increasing trends were previously reported from 2003 to 2007 in China, ${ }^{10}$ from 1975 to 2012 in the USA, ${ }^{9}$ and from 1980 to 2007 in Canada. ${ }^{19}$ These increasing trends might be associated with economic development and improvements in the diagnosis, and more exposure on risk factors worldwide. The rate of DALYs appeared to remain stable, while there was an increase in the absolute number of DALYs.

The age-standardised 5-year net survival rate of liver cancer was generally low $(10 \%-20 \%),{ }^{20}$ and only $10.1 \%$ in China. ${ }^{21}$ Furthermore, there have been few improvements in the survival rate of liver cancer from 1995 to 2014 in most countries. ${ }^{22}$ The 5 -year relative survival rates were $10.7 \%$ for those diagnosed at a regional stage and $2.8 \%$ for those diagnosed at a distant stage. ${ }^{23}$ Due to the 

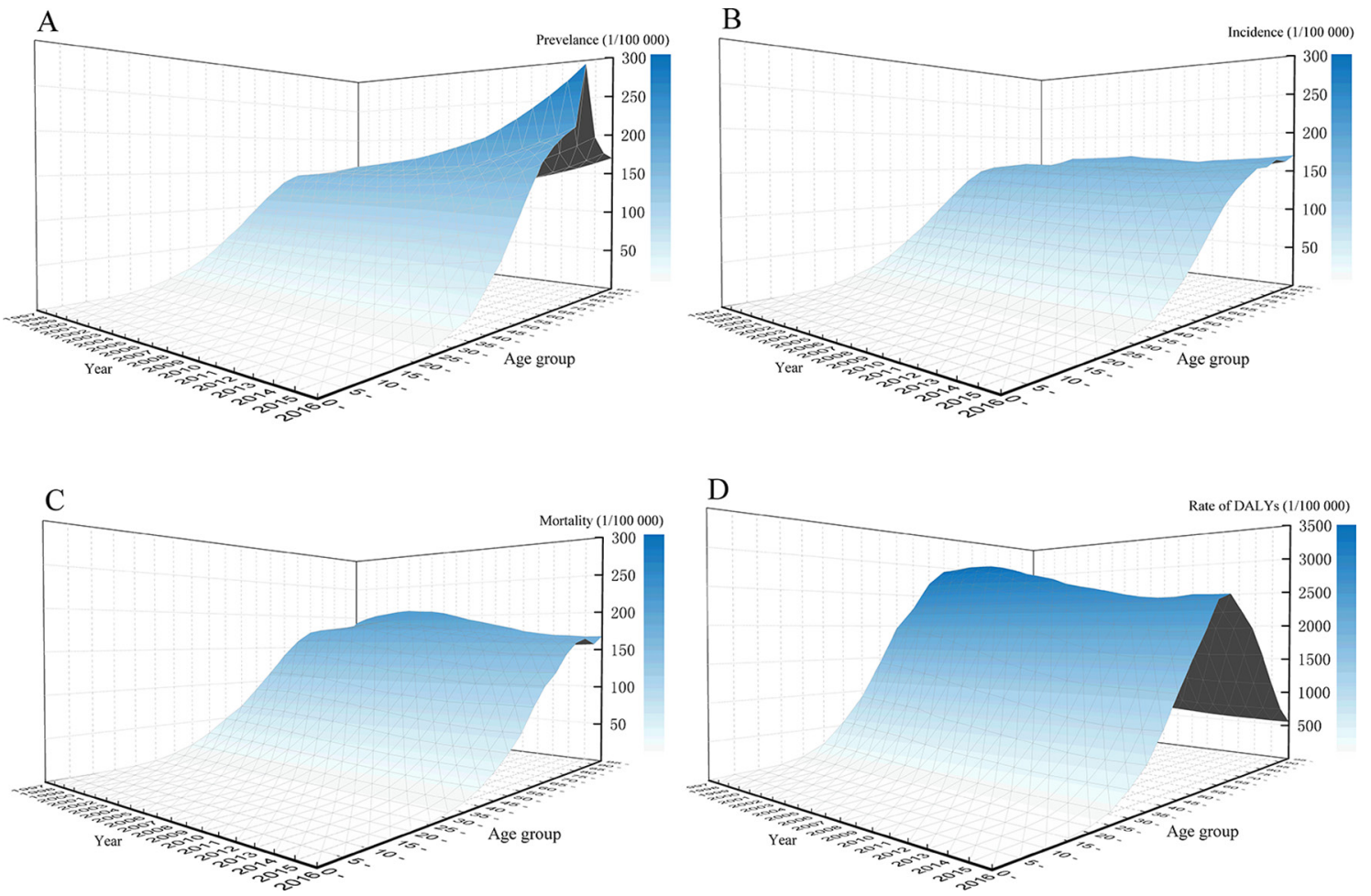

Figure 3 Disease burden of liver cancer among different age groups from 1997 to 2016 in China. (A) The changes in prevalence of liver cancer among different age groups; (B) the changes in incidence of liver cancer among different age groups; (C) the changes in mortality of liver cancer among different age groups; (D) the changes in rates of DALYs for liver cancer among different age groups. DALYs, disability-adjusted life years.

difficulties of making an early diagnosis, most patients were diagnosed at an advanced stage of the disease. ${ }^{24}$ Along with poor survival rates, the MI ratio of liver cancer was as high as $0.95^{325}$ indicating that the number of deaths were very close to the number of new cases and that the disease is difficult to cure. In China, the MI ratios were reported to be between 0.91 and 0.97 during $2005-$ $2009 .{ }^{26}$ The ratios observed in our study remained high, but a decreasing trend was observed. This may due to the improvements in medical care. For example, liver transplantation is the optimal for liver cancer. The number of

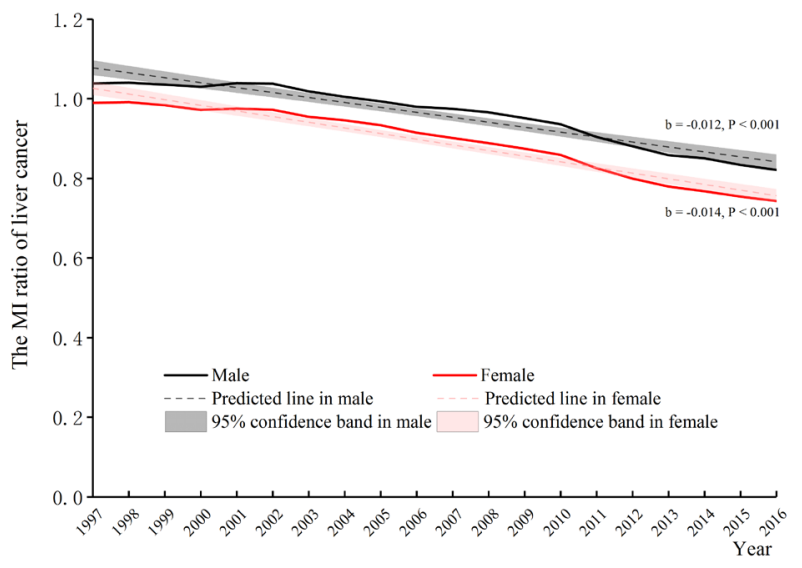

Figure 4 Changes in mortality-to-incidence (MI) ratio of liver cancer among different genders from 1997 to 2016 in China. liver transplantation increased from 1993 to 2013 (totally more than 24 000) in China. Additionally, the 5-year survival rate among patients was as high as $80 \%{ }^{27}$

\section{Factors associated with liver cancer}

The incidence of liver cancer was different between males and females except in the youngest age group (0-14 years old). ${ }^{3}$ Previous studies observed sex-related disparities in incidence and found rates in males that were twofold to threefold higher than those observed in females, ${ }^{925} 28$ and this difference was greater in high-incidence regions. ${ }^{6}$ Males experienced a two-fold or higher risk than females across all metrics included in our study, and these disparities may be exacerbated by other characteristics. For example, higher positive rates of hepatitis B surface antigen ( $\mathrm{HBsAg}$ ) were found in males than in females from multiple serological surveys across China, and the male to female ratio of HBsAg positive rate showed increasing $\left(10.30 \%\right.$ vs $7.29 \%$ in $1979^{29}$ and $8.6 \%$ vs $5.7 \%$ in $\left.2006^{30}\right)$. Males also had higher rates of drinking and alcohol consumption than females. ${ }^{31}{ }^{32}$ The male to female ratio of drinking $(61.3 \%$ vs $11.6 \%$ in 1993 and $38.3 \%$ vs $8.1 \%$ in 2006$)$ and alcohol intake $(28.0 \mathrm{~g} / \mathrm{d}$ vs. $12.9 \mathrm{~g} / \mathrm{d}$ in 1997 and $33.1 \mathrm{~g} / \mathrm{d}$ vs. $13.1 \mathrm{~g} / \mathrm{d}$ in 2006) both showed increasing in adults. ${ }^{33}$ The above evidence could strengthen the explanations on the increasing male to female ratio among all metrics in this study.

Cancer has a strong relationship with age, ${ }^{3}$ and the highest rates across all metrics were observed among those 
aged over 60 in our study. This may be attributed to the slow progression of the disease as the whole course from initiation to the development of clinically detectable liver cancer is estimated to take 5-20 years. ${ }^{34}$ The mean ages of diagnosed patients increased from 57.14 to 60.34 in males and 61.69 to 66.47 in females from 1989 to $2008 .{ }^{28}$ Additionally, the 5-year relative survival rate decreased from $38.8 \%$ among those aged under 45 to only $7.9 \%$ among those aged over $75 .^{23}$

In this study, we found that the age-adjusted trends of incidence, mortality and rate of DALYs did not showed increasing, only the prevalence in the recent years was still increasing after age adjustment. Actually, the crude rates of prevalence, incidence and mortality presented increasing trends. As the rates were adjusted by the population of 2010, the trends could be influenced due to the significant change of age structure from 1997 to 2016. The ageing of the population in China could contribute to the increasing disease burden of liver cancer. The population of mainland China reached nearly 1.39 billion at the end of 2016. And the proportions of those aged over 60 and over 65 accounted for $16.7 \%$ and $10.8 \%$ of the population, respectively, ${ }^{35}$ which is higher than the proportions of $11.96 \%$ and $8.24 \%$ of the $\mathrm{WHO}$ world standard population. ${ }^{36}$ Consistent with previous reports, our study found that the proportion of those aged over 60 had risen from $9.5 \%$ in 1997 to $16.1 \%$ in 2016. Meanwhile, the proportion of those aged below 15 decreased from $25.7 \%$ to $15.1 \%$.

Chronic infection with the hepatitis B virus (HBV) is one of the most important causes of liver cancer worldwide, especially in regions with highly endemic of $\mathrm{HBV}^{3}$ Liver cancer typically develops after 25-30 years of HBV infection, ${ }^{37}$ and individuals with a chronic HBV infection are 20-100 times more likely to develop liver cancer than uninfected individuals. ${ }^{38}$ In China, the weighted HBsAg-positive rate was $7.2 \%^{30}$ which led to China being categorised as a high-intermediate HBV endemic area. ${ }^{39}$ Though there is a negative trend in the infection rate in China, ${ }^{30}$ the number of HBV infections was still high due to the large population. The hepatitis $\mathrm{C}$ virus (HCV) can infect the immune system and endothelial cells, leading to immune dysfunction and chronic dysregulated angiogenesis. ${ }^{40}$ There is a moderate association between HCV infections and liver cancer (OR: $4.28,95 \%$ CI 3.30 to 5.56$){ }^{41}$ The prevalence of anti-HCV is estimated to be approximately $1 \%$ in China. ${ }^{42}$ As a result, HCV may have already contributed to a large number of liver cancer cases along with HBV. In our study, the proportions of viral factors (hepatitis B and $\mathrm{C}$ ) in prevalence, incidence, mortality and DALYs were more than $60 \%$ and increased with age. The presence of these high-risk factors makes liver cancer a strong candidate for targeted prevention projects. A prior study concluded that the incidence of liver cancer had fallen dramatically as a result of promoting dietary interventions and implementing neonatal $\mathrm{HBV}$ vaccinations. ${ }^{43}$

Alcoholic beverages have previously been characterised as 'carcinogenic to humans'. ${ }^{4}$ In 2010, 
alcohol-attributable cancers were estimated to be responsible for 337400 deaths worldwide, predominantly among men. Liver cancer was the largest contributor to alcohol-attributable cancer deaths (responsible for 23.9\%) and the DALYs lost (responsible for $24.7 \%$ ). ${ }^{3}$ The mechanism by which alcohol consumption increases the risk of HCC is primarily through the development of cirrhosis. ${ }^{56}$ A meta-analysis suggested a moderately detrimental role of alcohol consumption on liver cancer risk, ${ }^{7}$ while heavy alcohol consumption of $>80 \mathrm{~g} / \mathrm{d}$ of ethanol for at least 5 years increased the risk of HCC by nearly fivefold. ${ }^{45}$ Our study showed that the percentages of alcohol consumption as a cause of liver cancer in terms of prevalence, incidence, mortality and DALYs were greater than $10 \%$. The per capita alcohol consumption ( $>15$ years of age) rose from less than $2 \mathrm{~L}$ in 1981 to approximately $6.7 \mathrm{~L}$ in 2010, and will reach $8.3 \mathrm{~L}$ in $2025 .{ }^{46}$ Collectively, alcohol consumption in China has increased over the past few decades leading to an increase in the risk of liver cancer.

\section{Prevention and control of liver cancer}

Approximately one-third of cancer deaths can be attributed to the five leading behavioural and dietary risks: high body mass index, low fruit and vegetable intake, lack of physical activity, tobacco use and alcohol use. ${ }^{1}$ Since there are no standard and effective screening methods available for liver cancer, ${ }^{47}$ prevention is the most effective way for controlling the disease. ${ }^{2}$ A previous study concluded that implementing $\mathrm{HBV}$ vaccination, controlling $\mathrm{HCV}$ transmission and reducing alcohol intake could avoid more than $90 \%$ of worldwide liver cancer cases. ${ }^{48}$ Hepatitis B immunisation to reduce the risk of liver cancer was also recommended by the WHO. ${ }^{12}$ China had established an immunisation plan for hepatitis B in 1992 and has partnered with the Global Alliance on Vaccine and Immunisation since 2002. ${ }^{49}$ Strategies to address the other well-established risk factors such as promoting widespread public education, interrupting mother-to-infant transmission, monitoring of blood donors and blood products, improving the proportions of the age structure and implementing annual surveillance should be prioritised as well. ${ }^{42}$

\section{LIMITATIONS}

Although the GBD 2016 provides the most comprehensive and consistent data for assessments of global descriptive epidemiology, the general limitations of the GBD approach have been described. ${ }^{13-17}$ Descriptive results may differ from reports by local raw data, especially for estimates that depended on sophisticated statistical modelling to address sparse and inconsistent data. Meanwhile, data collection and model selection may cause errors, and which may be addressed in future iterations by the GBD study. Additionally, our study does not include estimates of the subnational (provincial or regional) or other potential factors (economic status, non-alcoholic fatty liver disease, obesity and smoking). Analysis on birth-cohort effects and others were not involved.
Better primary data from a national wide-coverage observational study or cancer registry on liver cancer burden are needed in the future.

\section{CONCLUSIONS}

China has made great efforts to shed its status as a 'leader in liver diseases' and achieved improvements in prevention, diagnosis, management and therapy for liver diseases in past decades. Despite these improvements, there remains a heavy disease burden that will increase if no effective interventions are implemented. Males and the elderly people exhibited the greatest, and overall, viral factors were the predominant causes of liver cancer. Despite a great deal of progress, the limitations of available therapies mean that prevention is the most effective approach to combat deadly liver cancer.

Contributors SY, LL and CD conceived and designed the study. CD, SY, YZ, XL, $\mathrm{JW}, \mathrm{CH}, \mathrm{MD}$ and $\mathrm{YL}$ extracted, cleaned, analysed the data and revised the paper critically. CD and XF wrote the first draft of the paper, contributed to figures and paper preparation. All authors critically revised the paper and gave final approval for publication. The corresponding author had full access to all the data in the study and had final responsibility for the decision to submit for publication.

Funding This work was supported by the National Natural Science Foundation of China, grant number [81672005, 81001271], the key joint project for data centre of the National Natural Science Foundation of China and Guangdong Provincial Government, grant number [U1611264], the Mega-Project of National Science and Technology of China, grant number [2018ZX10715014, 2013ZX10004904, 2014ZX10004008, 2013ZX10004901], Zhejiang Laboratory Project and the Fundamental Research Funds for the Central Universities.

Disclaimer The funders had no role in study design, data collection, data analysis or writing of the report.

Competing interests None declared.

Ethics approval Ethical approval was not obtained because the data included in this study were publicly available and de-identified data.

Provenance and peer review Not commissioned; externally peer reviewed. Data sharing statement No additional data are available.

Open access This is an open access article distributed in accordance with the Creative Commons Attribution Non Commercial (CC BY-NC 4.0) license, which permits others to distribute, remix, adapt, build upon this work non-commercially, and license their derivative works on different terms, provided the original work is properly cited, appropriate credit is given, any changes made indicated, and the use is non-commercial. See: http://creativecommons.org/licenses/by-nc/4.0/.

\section{REFERENCES}

1. World Health Organization. Cancer Fact sheet. http://www.who.int/ mediacentre/factsheets/fs297/en/ (accessed 30 Jan 2018).

2. Ferlay J, Soerjomataram I, Dikshit R, et al. Cancer incidence and mortality worldwide: sources, methods and major patterns in GLOBOCAN 2012. Int J Cancer 2015;136:E359-E386.

3. International Agency for Research on Cancer. World Cancer Report 2014. Geneva, Switzerland: WHO Press 2015.

4. Alzahrani B, Iseli TJ, Hebbard LW. Non-viral causes of liver cancer: does obesity led inflammation play a role? Cancer Lett 2014;345:223-9.

5. Hamed MA, Ali SA. Non-viral factors contributing to hepatocellular carcinoma. World J Hepatol 2013;5:311-22.

6. Chuang SC, La Vecchia C, Boffetta P. Liver cancer: descriptive epidemiology and risk factors other than HBV and HCV infection. Cancer Lett 2009;286:9-14.

7. Turati F, Galeone C, Rota M, et al. Alcohol and liver cancer: a systematic review and meta-analysis of prospective studies. Ann Oncol 2014;25:1526-35.

8. Chen JG, Zhang SW. Liver cancer epidemic in China: past, present and future. Semin Cancer Biol 2011;21:59-69. 
9. Ryerson AB, Eheman CR, Altekruse SF, et al. Annual Report to the Nation on the Status of Cancer, 1975-2012, featuring the increasing incidence of liver cancer. Cancer 2016;122:1312-37.

10. Chen JG, Chen WQ, Zhang SW, et al. [Incidence and mortality of liver cancer in China: an analysis on data from the National Registration System between 2003 and 2007]. Zhonghua Liu Xing Bing Xue Za Zhi 2012;33:547-53.

11. Chen W, Zheng R, Baade PD, et al. Cancer statistics in China, 2015. CA Cancer J Clin 2016;66:115-32.

12 .World Health Organization. Global action plan for the prevention and control of NCDs 2013-2020. Geneva, Switzerland: WHO Press, 2013.

13. Global Burden of Disease Collaborative Network. Global Burden of Disease Study 2016 (GBD 2016) Results. http://ghdx.healthdata.org/ gbd-results-tool (accessed 10 Jan 2018).

14. GBD 2016 DALYs and HALE Collaborators. Global, regional, and national disability-adjusted life-years (DALYs) for 333 diseases and injuries and healthy life expectancy (HALE) for 195 countries and territories, 1990-2016: a systematic analysis for the Global Burden of Disease Study 2016. Lancet 2017;390:1260-344.

15. Murray CJ, Ezzati M, Flaxman AD, et al. GBD 2010: design, definitions, and metrics. Lancet 2012;380:2063-6.

16. Murray CJ, Lopez AD. Measuring the global burden of disease. $N$ Engl J Med 2013;369:448-57.

17. GBD 2016 Disease and Injury Incidence and Prevalence Collaborators. Global, regional, and national incidence, prevalence, and years lived with disability for 328 diseases and injuries for 195 countries, 1990-2016: a systematic analysis for the Global Burden of Disease Study 2016. Lancet 2017;390:1211-59.

18. National Bureau of Statistics of the People's Republic of China. Tabulation on the 2010 population census of the People's Republic of China. http://www.stats.gov.cn/tjsj/pcsj/rkpc/6rp/indexch.htm (Accessed 23 Mar 2018).

19. De P, Dryer D, Otterstatter MC, et al. Canadian trends in liver cancer: a brief clinical and epidemiologic overview. Curr Oncol 2013;20:e40-3.

20. Allemani $\mathrm{C}$, Weir $\mathrm{HK}$, Carreira $\mathrm{H}$, et al. Global surveillance of cancer survival 1995-2009: analysis of individual data for 25,676,887 patients from 279 population-based registries in 67 countries (CONCORD-2). Lancet 2015;385:977-1010.

21. Zeng H, Zheng R, Guo Y, et al. Cancer survival in China, 2003-2005: a population-based study. Int J Cancer 2015;136:1921-30.

22. Allemani $\mathrm{C}$, Matsuda T, Di Carlo V, et al. Global surveillance of trends in cancer survival 2000-14 (CONCORD-3): analysis of individual records for 37513025 patients diagnosed with one of 18 cancers from 322 population-based registries in 71 countries. Lancet 2018;391:1023-75.

23. Howlader N, Noone A, Krapcho M, et al. SEER Cancer Statistics Review, 1975-2014 [based on November 2016 SEER data submission, posted to the SEER web site, April 2017]. https://seer. cancer.gov/csr/1975 2014/ (Accessed 30 Jan 2018).

24. Fu J, Wang $\mathrm{H}$. Precision diagnosis and treatment of liver cancer in China. Cancer Lett 2018;412:283-8.

25. Petrick JL, Braunlin M, Laversanne M, et al. International trends in liver cancer incidence, overall and by histologic subtype, 1978-2007. Int J Cancer 2016;139:1534-45.

26. Jiang N, Li S, Yan Y, et al. Trends of disease burden of liver cancer in Chinese cancer registries, 2005-2009. Modern Preventive Medicine 2017;44:3483-7.

27. Shusen Z, Jun YU, Wu Z. Current development of liver transplantation in China. J Clin Hepatol 2014;30:2-4.

28. Zhang $\mathrm{S}$, Zheng $\mathrm{R}$, Zeng $\mathrm{H}$, et al. [The incidence differences among sex, geographical areas and mean age of diagnosis for liver cancer in China, 1989-2008]. Zhonghua Yu Fang Yi Xue Za Zhi 2014;48:355-60.

29. Chongbai L. Epidemiological characteristics and epidemiological factors of viral hepatitis in China. Chin J Hepatol 1998;6:67-70.

30. Liang X, Bi S, Yang W, et al. Reprint of: Epidemiological serosurvey of Hepatitis B in China--declining HBV prevalence due to Hepatitis B vaccination. Vaccine 2013;31 Suppl 9(Suppl 9):J21-J28.

31. Li $\mathrm{Y}$, Jiang $\mathrm{Y}$, Zhang $\mathrm{M}$, et al. Drinking behaviour among men and women in China: the 2007 China Chronic Disease and Risk Factor Surveillance. Addiction 2011;106:1946-56.

32. Millwood IY, Li L, Smith M, et al. Alcohol consumption in 0.5 million people from 10 diverse regions of China: prevalence, patterns and socio-demographic and health-related correlates. Int J Epidemiol 2013;42:816-27.

33. Ma YX, Zhang B, Wang HJ, et al. [Status and trend of alcohol consumption among adults in nine provinces (antonomous region) of China from 1993 to 2006]. Zhonghua Yu Fang Yi Xue Za Zhi 2011;45:323-9.

34. Moriwaki H. Prevention of liver cancer: current strategies and future perspectives. Int J Clin Oncol 2002;7:27-31.

35. National Bureau of Statistics of the People's Republic of China. Statistical Communique on National Economic and Social Development in 2016 http://www.stats.gov.cn/tjsj/zxfb/201702/ t20170228_1467424.html (Accessed 5 Feb 2018).

36. Ahmad OB, Boschi-Pinto C, Lopez AD, et al. Age Standardization of Rates: A New WHO Standard. Gpe Discussion Paper 2001;31:12.

37. Lee WM. Hepatitis B virus infection. N Engl J Med 1997;337:1733-45.

38. Chu CM. Natural history of chronic hepatitis B virus infection in adults with emphasis on the occurrence of cirrhosis and hepatocellular carcinoma. J Gastroenterol Hepatol 2000;15 Suppl:E25-E30.

39. Ott JJ, Stevens GA, Groeger J, et al. Global epidemiology of hepatitis B virus infection: new estimates of age-specific $\mathrm{HBsAg}$ seroprevalence and endemicity. Vaccine 2012;30:2212-9.

40. Ali N, Allam H, May R, et al. Hepatitis C virus-induced cancer stem cell-like signatures in cell culture and murine tumor xenografts. $J$ Virol 2011;85:12292-303.

41. Luo RH, Zhao ZX, Zhou XY, et al. Risk factors for primary liver carcinoma in Chinese population. World J Gastroenterol 2005;11:4431-4.

42. Cui Y, Jia J. Update on epidemiology of hepatitis B and $C$ in China. $J$ Gastroenterol Hepatol 2013;28 Suppl 1(Suppl 1):7-10.

43. Sun Z, Chen T, Thorgeirsson SS, et al. Dramatic reduction of liver cancer incidence in young adults: 28 year follow-up of etiological interventions in an endemic area of China. Carcinogenesis 2013;34:1800-5.

44. Grewal P, Viswanathen VA. Liver cancer and alcohol. Clin Liver Dis 2012;16:839-50.

45. Donato F, Tagger A, Gelatti U, et al. Alcohol and hepatocellular carcinoma: the effect of lifetime intake and hepatitis virus infections in men and women. Am J Epidemiol 2002;155:323-31.

46. World Health Organization. Global status report on alcohol and health 2014. Geneva, Switzerland: WHO Press, 2014.

47. Vogtmann E, Shu XO, Li HL, et al. Cholelithiasis and the risk of liver cancer: results from cohort studies of 134,546 Chinese men and women. J Epidemiol Community Health 2014;68:565-70.

48. La Vecchia C. Cancer and liver cancer prevention: is it a fact or just a potential? Hepatology 2008;48:7-9.

49. Cui F, Li L, Hadler SC, et al. Factors associated with effectiveness of the first dose of hepatitis B vaccine in China: 1992-2005. Vaccine 2010;28:5973-8. 